BULL. AUSTRAL. MATH. SOC.

VOL. 28 (1983), 355-365.

\title{
FINITE SIMPLE GROUPS AND FINITE PRIMITIVE PERMUTATION GROUPS
}

\author{
Cherry E. Praeger
}

\begin{abstract}
The classification of the finite simple groups has had farreaching consequences for many branches of algebra. This paper is a discussion of several problems about primitive permutation groups which have been solved using the simple group classification.
\end{abstract}

\section{Introduction}

The central problem of the theory of finite groups is to find for each positive integer $n$ all groups (up to isomorphism) of order $n$. This is of course equivalent to the two problems of

(i) finding all finite simple groups and

(ii) finding all extensions of one group by another group.

The solution to the more basic problem of classifying all finite simple groups was completed in 1980 and it is now the job of mathematicians in related fields to determine the consequences of this classification on their field of research. The purpose of this paper is to survey several fairly basic problems in the theory of finite permutation groups where the simple group classification has led to a satisfactory solution.

Received 19 July 1983. This paper is based on an invited lecture given at the 1983 Australian Mathematical Society Annual Meeting held at the University of Queensland in May 1983.

Copyright Clearance Centre, Inc. Serial-fee code: 0004-9727/83 $\$ A 2.00+0.00$. 
As an analogue of the preceding discussion of finite groups one might say that the central problem of the theory of finite permutation groups is to find for each positive integer $n$ all permutation groups (up to equivalence) of degree $n$. (A permutation group of degree $n$ is a subgroup of the symmetric group $S_{n}$ of all permutations of the set $\mathbf{n}=\{1,2, \ldots, n\}$. Two permutation groups $G, H$ of degree $n$ are said to be equivalent if there is a group isomorphism $f: G \rightarrow H$ and a bijection $\phi: n \rightarrow n$ such that for all $g$ in $G$ and $i$ in $n$, $\left(i^{g}\right) \phi=(i \phi)^{g f}$; that is $G$ and $H$ are equivalent if and only if they are isomorphic and act in the same way on $n$ up to a relabeliing of $n$.) Now let $G \leq S_{n}$ : then $G$ determines an equivalence relation on $n$ by

$$
i \sim j \Leftrightarrow i^{g}=j \text { for some } g \text { in } G \text {. }
$$

The equivalence classes are called orbits and $G$ is called transitive if it has exactly one orbit. The central problem is then equivalent to finding

(i) all transitive permutation groups and

(ii) all ways of putting together transitive groups to find all permutation groups.

So let us now suppose that $G \leq S_{n}$ is transitive. Sometimes it is possible for $n$ to be partitioned into blocks of equal size which are permuted (blockwise) by $G$ (that is for such a block $B$ and $g$ in $G$, $B^{g}$ either equals $B$ or is disjoint from $B$ ). $G$ is said to be primitive if the only possible sets of blocks are $\{n\}$ and $\{\{i\} \mid 1 \leq i \leq n\}$. The problem of finding all transitive permutation groups is then equivalent to finding

(i) all primitive permutation groups and

(ii) all ways of putting together primitive groups to find all transitive groups.

While problem (ii) is by no means trivial, the problem of finding all primitive groups is more basic to the subject, and indeed the primitive permutation groups play a role in the theory of finite permutation groups 
analogous to the role of finite simple groups in the theory of finite groups. Certainly the consequences of the simple group classification have been most spectacular for problems about primitive permutation groups.

\section{Simple groups and primitive groups}

The finite simple groups $G$ may be conveniently listed as follows:

(a) $G=Z_{p}$, the cyclic group of order $p, p$ a prime;

(b) $G=A_{n}$, the alternating group of degree $n$, that is the group of all even permutations of a set of size $n, n \geq 5$;

(c) $G$ a group of Lie type: these may be divided into

(i) $G=G(n, q)$, a classical group of dimension $n$ "over" a field of $q$ elements (these comprise linear, orthogonal, unitary and symplectic groups, six families in all),

(ii) $G=G(q)$, an exceptional group over a field of $q$ elements (10 families);

(d) $G$ is one of the 26 sporadic simple groups.

Further information about groups of Lie type may be found in [9] for example, and about the sporadic simple groups in [10].

So far, the most useful result which has allowed the classification of the finite simple groups to be used to make headway with problems involving primitive permutation groups is a theorem of M. O'Nan and L.L. Scott. It allows some questions about primitive permutation groups $G$ to be reduced to the cases where $G$ is a group of affine transformations of a vector space or $T \leq G \leq$ Aut $T$ for some non-abelian simple group $T$. In essence their result is as follows (see [6], Theorem 4.1, for details and [1], [12] for details of the correction at part (ii)).

THEOREM 2.1. Let $G$ be a primitive permutation group on a set $X$ of $n$ points, and let $N$ be the socle of $G$ (that is the group generated by all minimal normal subgroups of $G$ ). Then one of the following occurs.

(i) $N$ is elementary abelian of order $p^{d}$ and regular, $n=p^{d}$ where $p$ is prime and $d \geq 1$, and $G \leq \operatorname{AGL}(d, p)$ the group of affine 
transformations of $N$.

(ii) $N=T_{1} \times \ldots \times T_{m}$ where $T_{i}$ are isomorphic to a fixed nonabelian simple group $T$ and $m \geq 1$. Moreover if $m \geq 2$ then

(a) $n=|T|^{m-1}$ and the action of $N$ on $X$ is equivalent to $i$ ts "diagonal action" on the cosets of a diagonal subgroup $D=T$ of $N$, or

(b) $n=n_{0}^{k}, m=k r, G \leq G_{0}$ wr $S_{k}$, where $G_{0}$ is primitive on $Y$ of degree $n_{0}$ with a minimal normal subgroup isomorphic to $T^{r}$, and the action of $G$ on $X$ is equivalent to its "product action" on $Y^{k}$. Further either $T^{r}$ is the socle of $G_{0}$ (and $G_{0}$ satisfies (a) or $r=1$ ), or $r=1$ and $T$ is reguiar on $Y$.

Of course other links exist between certain questions about primitive groups and related questions about simple groups, but this theorem has proved the most useful. We shall now consider several longstanding problems about primitive permutation groups for which the classification of simple groups has provided major "break throughs".

\section{How big are primitive permutation groups?}

The symmetric group $S_{n}$ and the alternating group $A_{n}(n \neq 2)$, are both primitive of degree $n$ and $\left|S_{n}\right|=2\left|A_{n}\right|=n !$. The problem of finding an upper bound $f(n)$, "much smaller" than $n !$, for the orders of primitive groups $G$ of degree $n$ other than $S_{n}$ and $A_{n}$ was one of the central problems of 19 th century group theory. The best result obtained last century is the theorem of Bochert ([5], or see [21], 14.2) that $f(n)=n ! /[(n+1) / 2]$ ! is an upper bound. No significant progress was made until 1969 when Wielandt [22] showed that for primitive but not doubly transitive groups, $f(n)$ could be taken as $24^{n}$. This result was generalised in 1981 to all primitive groups with $f(n)=4^{n}$, by Saxl and the author [18]. Soon after this and using completely different methods 
Babai [2, 3] showed that $f(n)=\exp \left(4 V_{n} \log ^{2} n\right)$ was a bound. These various bounds can be compared easily using the following table, where $(\log f(n))^{*}$ denotes the order of the dominant term in the asymptotic expansion of $\log f(n)$. However using the classification of simple groups Cameron ([6], Theorem 6.1 (s)) showed that $f(n)$ could be taken as $n^{c \log n}$ for some constant $c$, if one excludes $G \leq S_{m}$ wr $S_{k}$ in the product action, where $S_{m}$ is acting on $j$-element subsets, $j \geq 1$, $k \geq 1$.

TABLE 1. Orders of prinitive groups

\begin{tabular}{|l|c|c|c|}
\hline \multicolumn{2}{|c|}{} & $f(n)$ & $(\log f(n))^{*}$ \\
\hline $\begin{array}{l}\text { Wochert } \\
\text { Praeger, Saxi }\end{array}$ & $\begin{array}{l}1889 \\
1969 \\
1981\end{array}$ & $n ! /[(n+1) / 2] !$ & $n \log n$ \\
Babai & 1982 & $4^{n}$ & $n$ \\
\hline $\begin{array}{l}\text { Cameron (I) } \\
\text { Babai, Cameron } \\
\text { Palfy (1) }\end{array}$ & 1981 & $n^{c \log n}$ & $\sqrt{n} \log ^{2} n$ \\
\hline
\end{tabular}

(I) Bound for a subclass of primitive groups, see text.

He showed that even better results were possible if one was "careful". Finally (also using the simple group classification) Babai, Cameron and Palfy [4] showed that if the set of composition factors of the primitive group $G$ of degree $n$ contains no alternating group of degree greater than $d$ and no classical group of dimension greater than $d$ for some fixed positive integer $d$ then $|G|$ is polynomially bounded, that is $|G| \leq n^{c}$ for some constant $c=c(d)$.

\section{How many primitive permutation groups are there?}

For each positive integer $n$ the symmetric group $S_{n}$ is primitive of degree $n$, as is the alternating group $A_{n}$ for $n \neq 2$, while, for $n \leq 4$, there are no other primitive groups of degree $n$. By 1861 Mathieu 
[14] had shown that for each $n=5, \ldots, 33$ there was a primitive (indeed a multiply transitive) group of degree $n$ other than $A_{n}$ and $S_{n}$.

Let $E$ be the set of positive integers $n$ for which there exists a primitive group of degree $n$ other than $A_{n}$ and $S_{n}$. Thus $E$ contains \{n $\mid 5 \leq n \leq 33\}$. It is non-trivial to show that $E$ does not contain 34. In 1970 Sims [19] published a complete list of primitive groups of degrees $n \leq 20$, and he has determined (but not published) the primitive groups of degrees $n \leq 50$ : the only integers $n, 5 \leq n \leq 50$, which are not in $E$ are 34,39 and 46 . Further, certain other integers were proved to lie outside of $E$ by Miyamoto [15] in 1975 and Neumann and SaxI [16] in 1979: this was a consequence of their investigations of primitive groups of certain special degrees, for example

(i) if $n=2 p=4 q+2=f(r)>22, p, q, r$ prime, $f(r)=r+3, r+5$ or $5 r+3$ then $n \notin E$ (for example, $n=46,94,118,166,214, \ldots)$,

(ii) if $n=4 p$ where $p$ and $(p-1) / 2$ are prime and $4 p-1$ is composite then $n \notin E$ (for example $n=92,188,236, \ldots)$.

These "pre-classification" results strongly suggest that $\mathbb{N}-E$ is infinite. However using the simple group classification Cameron, Neumann and Teague [7] have shown not only that $N-E$ is infinite but also that $E$ has density zero in the natural numbers $N$, that is that non-trivial primitive groups $G$ of degree $n$ (that is $G \neq S_{n}, A_{n}$ ) are rare. This is a very surprising result which had not even been guessed at before the classification. If $e(x)=|\{n \in E \mid n \leq x\}|$, then it is shown that

$$
\begin{aligned}
e(x) & =2 \pi(x)+(1+\sqrt{2}) x^{\frac{1}{2}}+o\left(x^{\frac{1}{2}} / \log x\right) \\
& \sim 2 x / \log x .
\end{aligned}
$$

That $e(x) \geq 2 \pi(x)+(1+\sqrt{2}) x^{\frac{1}{2}}-o(\log x)$ follows easily from the facts that $n=p \in E, p$ a prime (taking $G=z_{p}$ ), $n=p+1 \in E$, $\left(G=\operatorname{PSL}(2, p)\right.$ in its natural action on the projective line), $n=m^{2} \in E$ $\left(G=S_{m}\right.$ wr $Z_{2}$ in its product action), $n=m(m-1) / 2 \in E \quad\left(G=S_{m}\right.$ on unordered pairs) and there are at most $O(\log x)$ cases where these 
integers coincide. The classification of simple groups is used to show that $e(x)$ is at most the expression above. This involves an examination of permutation representations of alternating groups and groups of lie type.

\section{Which permutations are excluded from primitive groups?}

Let $G$ be a primitive permutation group of degree $n, G \neq S_{n}, A_{n}$. We have seen that $|G|$ is much smaller than $n !$. Are there many permutations $g \in S_{n}$ which are excluded from membership of any such $G$ ? In 1873 Jordan [11] showed that any permutation of prime order $p$ with exactly one cycle of length $p$ and at least 3 fixed points is excluded from membership of any primitive $G\left(\neq S_{n}, A_{n}\right)$.

A permutation of $g \in S_{n}$ is said to have type

$$
t=1^{a_{1} a_{2}} \ldots n^{a_{n}}, \sum i a_{i}=n
$$

if $g$ has $a_{i}$ cycles of length $i$ for $i=1, \ldots, n$. Let $t_{n}$ be the set of types $t$ of permutations in $S_{n}$ such that no permutation of type $t$ may belong to any primitive permutation group $G$ of degree $n$, $G \neq S_{n}, A_{n}$. Then Jordan's result is that $t=1^{a} p^{1} \in T_{n}$ where $p$ is prime and $a=n-p \geq 3$. Of course the previous section shows that $T_{n}$ contains all types for "almost all" $n$ since for almost all $n$ no nontrivial primitive groups exist. Nevertheless the sets $T_{n}$ are very much of interest as long as the classification of primitive permutation groups is incomplete. Jordan announced similar results, namely that $1^{a} p^{q} \in T_{n}$ where $p$ is prime $p>q, 1 \leq q \leq 5$ and $a>q+1$. Proofs of these results did not appear until W.A. Manning published them in 1909 and 1915. The best result of this period was obtained by Manning in 1918 (see [17] for a summary of results of the time), namely

$$
\mathrm{t}=1^{a} p^{q} \in T_{n}, p \text { prime, } 5<q \leq(p+1) / 2, a>4 q-4 .
$$

There were efforts to extend the range of $q$, but the lower bound on $a$ 
had to increase markedly since the groups $S_{m}$ and $A_{m}$, $m=q+(p+1) / 2$, acting on unordered pairs contain an element of type ${ }_{1} a^{q}$ with $a=\left((2 q-p)^{2}-1\right) / 8$. In 1979, [17], it was shown that these were the only groups containing such elements; namely if $2<q<p$ and $a>(5 q-4) / 2$ then either $t=1^{a} p^{q} \in T_{n}$, or $n=m(m-1) / 2$, where $m=q+(p+1) / 2, \quad a=\left((2 q-p)^{2}-1\right) / 8$, and in this case $A_{m}$ and $S_{m}$ on unordered pairs are the only non-trivial primitive groups containing elements of type $1^{a} p^{q}$.

Very recently using the simple group classification Liebeck and Saxl [13] have been able to classify all primitive permutation groups which contain elements of type ${ }_{1}^{a} p^{q}$ for $q<p$ and any $a \geq 0$.

\section{Primitive groups with given rank}

Let $G \leq S_{n}$ be primitive and let $X_{1}=\{1\}, X_{2}, \ldots, X_{r}$ be the orbits of the stabilizer $G_{1}$ of 1 in $n$. Then $G$ is said to have rank $r$. Clearly $r \geq 2$ and if $r=2, G$ is called 2-transitive. One of the first major consequences of the classification of the simple groups was the classification of all finite 2-transitive groups. A proof of this important result is given in Cameron's paper [6], Theorem 5.3 (S). In his paper various other results about groups with given rank are discussed.

\section{Primitive groups with given subdegree}

With the notation of the previous section, the integers $d_{i}=\left|x_{i}\right|$, $I \leq i \leq r$, are called the subdegrees of $G$. Let $d$ be one of the subdegrees. It was conjectured by sims that the order of $G_{1}$ is bounded by a function of $d$. That $G$ is primitive is crucial for the conjecture to be true since the usual imprimitive action of $G=S_{(d+1)}$ wr $S_{k}$ of degree $n=(d+1) k$ has a subdegree $d$ and the stabilizer of a point has order $d !(d+1) !^{(k-1)}(k-1) !$. This conjecture has been much studied in the past 15 
years and many partial results have been obtained. In particular Thompson [20] showed that $G_{1}$ has a normal subgroup of prime power order whose index in $G_{1}$ is bounded by a function of $d$. It has been possible to use the classification together with Thompson's result to prove the conjecture true. Although no attempt was made to get the best possible function it was shown that $\left|G_{1}\right| \leq \exp \left(d^{2} o(d)\right),[8]$.

\section{Closely related topics}

Permutation groups often arise as automorphism groups of geometrical structures, and in these related areas one would expect to find new theorems which rely on the simple group classification. Algebraic graph theory is one field where several results have been proved and many more can be expected - for example one consequence of Sims' conjecture is that there are only a finite number of distance transitive graphs with a given valency, see [8]. In the theory of designs, already the 2-transitive designs have been classified (by W.M. Kantor (private communication)), and I would expect more results in this area also in the near future.

\section{References}

[1] M. Aschbacher and J.L. Scott, "Maximal subgroups of finite groups", J. Algebra (to appear).

[2] L. Babai, "On the order of uniprimitive permutation groups", Ann. of Math. (2) 113 (1981), 553-568.

[3] L. Babai, "On the order of doubly transitive permutation groups", Invent. Math. 65 (1982), 473-484.

[4] L. Babai, P.J. Cameron and P.P. Palfy, "On the orders of primitive groups with restricted nonabelian composition factors", $J$. Algebra 79 (1982), 161-168.

[5] A. Bochert, "Ueber die Zahl der verschiedenen Werthe, die eine Function gegebener Buchstaben durch Vertauschung derselben erlangen kann", Math. Ann. 33 (1889), 584-590. 
[6] P.J. Cameron, "Finite permutation groups and finite simple groups", Bulz. London Math. Soc. 13 (1981), 1-22.

[7] P.J. Cameron, P.M. Neumann and D.N. Teague, "On the degrees of primitive permutation groups", Math. Z. 180 (1982), 141-149.

[8] P.J. Cameron, C.E. Praeger, J. Saxl and G.M. Seitz, "On the sims conjecture and distance transitive graphs", Bull. London Math. Soc. 15 (1983), 499-506.

[9] R.W. Carter, Simple groups of Lie type (John Wiley \& Sons, London, New York, Sydney, 1972).

[10] J.F. Hurley and A. Rudvalis, "Finite simple groups", Amer. Math. Monthly 84 (1978), 693-714.

[11] C. Jordan, "Sur la limite de transitivité des groupes non alternés", Bulz. Soc. Math. France 1 (1873), 40-71.

[12] L.G. Kovács, "Maximal subgroups in composite finite groups", in preparation.

[13] M. Liebeck and J. Saxl, "Primitive permutation groups containing an element of large prime order", unpublished.

[14] E. Mathieu, "Mémoire sur l'étude des fonctions de plusieurs quantités, sur le manière de les former et sur les substitutions qui les laissent invariables", J. Math. Pure Appl. (Liouville) (2) $6(1861), 241-323$.

[15] 1. Miyamoto, "On primitive permutation groups of degree $2 p=4 q+2, p$ and $q$ being prime numbers", J. Fac. Sci. Univ. Tokyo Sect. IA Math. 22 (1975), 17-23.

[16] P.M. Neumann and J. Saxl, "The primitive permutation groups of some special degrees, II: small multiples of certain large primes", Math. Z. 169 (1979), 205-222.

[17] C.E. Praeger, "On elements of prime order in primitive permutation groups", J. Algebra 60 (1979), 126-157.

[18] C.E. Praeger and J. SaxI, "On the orders of primitive permutation groups", Bull. London Math. Soc. 12 (1980), 303-307. 
[19] C.C. Sims, "Computational methods in the study of permutation groups", Computational problems in abstract algebra, 169-183 (Proc. Conf. Oxford, 1967. Pergamon, London, 1970).

[20] J.G. Thompson, "Bounds for orders of maximal subgroups", J. AZgebra 14 (1970), 135-138.

[21] H. Wielandt, Finite permutation groups (Academic Press, New York, London, 1964 ).

[22] H. Wielandt, Permutation groups through invariant relations and invariant fronctions (Ohio State University Lecture Notes. Ohio State University, Columbus, Ohio, 1969).

Department of Mathematics, University of Western Australia, Nedlands, Western Australia 6009, Australia. 